\title{
Photoelectron spectra and high Rydberg states of lithium generated by intense lasers in the over-the-barrier ionization regime
}

\author{
Toru Morishita ${ }^{1}$ and C. D. Lin $^{2}$ \\ ${ }^{1}$ Department of Engineering Science, The University of Electro-Communications, 1-5-1 Chofu-ga-oka, Chofu-shi, Tokyo 182-8585, Japan \\ ${ }^{2}$ J. R. Macdonald Laboratory, Physics Department, Kansas State University, Manhattan, Kansas 66506-2604, USA
}

(Received 22 April 2013; published 6 June 2013)

\begin{abstract}
We calculated photoelectron energy and momentum spectra and the population of high Rydberg states of lithium atoms by intense $785 \mathrm{~nm}$ laser pulses at intensities in the over-the-barrier ionization (OBI) regime. The calculated spectra are compared to experiments reported in Schuricke et al. [Phys. Rev. A 83, 023413 (2011)]. It is shown that in the OBI regime, due to strong depletion of the ground state, the photoelectron spectra are generated from the leading edge of the laser pulse only, resulting in spectra that are nearly independent of laser intensities. Analysis of the calculated spectra reveals that total ionization probability is suppressed as the intensity is increased in the OBI regime. The suppression is due to the increase of excitation probability of high Rydberg states in the OBI regime, demonstrating that atoms and molecules are never fully ionized at high intensities. We also conclude that the interference stabilization model is not needed to explain the formation of high Rydberg states, and that there is no evidence that laser-dressed Kramers-Henneberger states play a role in the strong-field ionization of atoms and molecules by infrared lasers in the OBI regime.
\end{abstract}

DOI: 10.1103/PhysRevA.87.063405

PACS number(s): $32.80 . \mathrm{Rm}$, 42.65.Ky

\section{INTRODUCTION}

In the past two decades, strong-field ionization of atomic targets has been studied widely both in theoretical calculations and in experiments [1-9]. Most of the experiments have focused on rare-gas atoms whose ionization potential $I_{p}=$ $12-25 \mathrm{eV}$, with the laser wavelength centered around $800 \mathrm{~nm}$. For such measurements, it takes about ten or more photons to ionize the atom. In a few cases, experiments have been carried out at shorter wavelengths close to $600 \mathrm{~nm}$ [2,3]. Lately, midinfrared lasers have been used in ionization studies and a number of interesting low-energy structures in the electron spectra have been reported [10-12].

Strong-field ionization by low-frequency lasers is often discussed in terms of multiphoton ionization (MPI) or tunneling ionization (TI) models. In the weak-field regime, the perturbative MPI model would predict distinct equal-spaced sharp peaks in the photoelectron spectra [called above-threshold ionization (ATI)] where the spectral strength drops rapidly with the number of absorbed photons. On the other hand, in the strong-field regime, the TI model predicts a photoelectron spectrum that drops steeply from the threshold to around $2 U_{p}$, but then it flattens out between 2 and $10 U_{p}$. Here, $U_{p}$ is the ponderomotive energy of an electron in the laser field. These high-energy electrons arise from backscattering of laser-driven returning electrons with the target ion $[13,14]$. Such recollisions are also responsible for high-order harmonic generation and nonsequential double-ionization phenomena [15-17]. As the laser intensity is further increased, one enters the so-called over-the-barrier ionization (OBI) regime. In the OBI regime, a classical electron can leak out over the barrier formed by the combined potential from the atomic ion and the electric field of the laser. The threshold electric field is given by $I_{p}^{2} / 4$ in a.u. for an electron with ionization potential $I_{p}$. Note that this simple expression, which is commonly used in the literature, is derived by assuming the electron is moving in a one-dimensional Cartesian coordinate. A more precise estimate based on the parabolic coordinates [18] in three dimensions (3D) gives the threshold to be $\kappa^{4} /[8(2-\kappa)]$ a.u., where $\kappa=\sqrt{2 I_{p}}$. For easy distinction, this less familiar but more accurate threshold will be called the $\eta$-OBI threshold, in view of the fact that the potential barrier is formed in the $\eta$ parabolic coordinate.

The division of strong-field ionization of atoms and molecules by low-frequency infrared (IR) lasers in terms of Keldysh parameter $\gamma=\sqrt{I_{p} / 2 U_{p}}$, or in terms of MPI, TI, and OBI, is too simple. Both TI and OBI are static concepts. Recently, an adiabatic theory based on the asymptotic expansion of a small parameter $\epsilon$, defined by the ratio between atomic and laser time scales $\left(\epsilon=\omega / I_{p}\right.$ for a monochromatic field), has been developed [19-21]. In this theory, the nonperturbative TI (as well as OBI) model has meaning only in the "adiabatic regime," i.e., for $\epsilon$ close to zero. This latter condition is satisfied for rare-gas atoms, but not for atoms such as $\mathrm{Li}$ when they are exposed to $\sim 800 \mathrm{~nm}$ laser pulses. In a recent experiment by Schuricke et al. [4], photoelectron spectra of Li atoms by $785 \mathrm{~nm}, 30 \mathrm{fs}$ pulses with intensities between $10^{11}$ and $10^{14} \mathrm{~W} / \mathrm{cm}^{2}$ were reported. Due to the small $I_{p}=5.39 \mathrm{eV}$, absorption of only four photons will be able to ionize a $\mathrm{Li}$ atom. At the OBI $\left(\eta\right.$-OBI) threshold intensity of $3.4 \mathrm{TW} / \mathrm{cm}^{2}$ $\left(7.2 \mathrm{TW} / \mathrm{cm}^{2}\right)$, the Keldysh parameter is $\gamma=3.6(2.5)$, which is much larger than the typical dividing line (at $\gamma \sim 1$ ) of the TI regime for rare-gas atoms. In terms of adiabatic parameter, for $\mathrm{Li}, \epsilon=0.29$, which is just near the onset of the adiabatic regime. In fact, the optical period of a $785 \mathrm{~nm}$ laser is $2.7 \mathrm{fs}$, which is not much longer than the typical orbiting period (about 0.6 fs if the radius of $2 s$ orbital is taken to be 2.5 a.u.) of the Li $2 s$ electron to justify the use of the TI or the OBI pictures. Thus, in the experiment of Schuricke et al., we are faced with the question of how the ionization evolves from the MPI mechanism at low intensity to the high-intensity regime where ionization probability reaches near unity.

It is well known that strong-field ionization of an atom can also be formulated in the frame of the oscillating laser field, in the so-called Kramers-Henneberger (KH) frame. In the 1990s, extensive theoretical works by Gavrila and coworkers [22,23] 
showed that atoms become stable against high-frequency photons at high laser intensities $(\epsilon \gg 1)$ using the high-frequency Floquet theory (HFFT). The leading-order solution of this theory predicts the existence of a new class of stable bound states, called KH states, for an atom. In the next order, ionization of an atom in the stabilization regime can be calculated from the initial KH states. Despite intense investigations in the 1990s, no clear evidence of stabilization has been firmly established experimentally for the ionization of atoms from valence electrons. Still, stabilization of an atom in a strong laser field has been repeatedly addressed in recent years, in the form of experimental observation of neutrals at high laser intensities. In addition, recently, Morales et al. [24] claimed that photoelectron momentum spectra can be used to "image" the KH bound states for $\mathrm{K}$ atoms in intense $800 \mathrm{~nm}$ laser pulses. For this system, $\epsilon=0.36$, which is close to the Li studied here. We thus also examined the "existence" of KH states from our calculations.

The goal of the present study is twofold. Initially, we intended to understand the origin of the remaining difference between the experimental photoelectron spectra and the theoretical calculations reported in Schuricke et al. In this paper, two different theoretical approaches were used and they showed discrepancies between the two methods and with experiment. We comment that a similar study for this purpose has also been carried out by Jheng and Jiang [25] recently. Second, since the experiment reported photoelectron momentum spectra at intensities way beyond the OBI threshold, we decided to carry out a careful analysis of the observed photoelectron spectra and touch upon issues such as ionization suppression, populations of Rydberg states, and evidences of KH states.

The rest of this paper is organized as follows. In Sec. II, we briefly describe the computational methods and parameters used in the calculation. Section III presents our theoretical results and comparison with experimental photoelectron energy and momentum spectra. In Sec. IV, we analyze photoelectron energy and momentum spectra calculated at fixed peak laser intensities in order to understand the fine structures observed in the experiment. Section V analyzes the population of Rydberg states and the mechanism of their formation, especially in the $\eta$-OBI regime. In doing so, we concluded that there is no need to employ the so-called interference stabilization mechanism for the formation of Rydberg states at high laser intensities. This section also addresses the issue of the existence of $\mathrm{KH}$ states for ionization of $\mathrm{Li}$ in the deep OBI regime. A short summary is given at the end to conclude.

\section{THEORETICAL METHODS}

We solved the time-dependent Schrödinger equation (TDSE) for a $\mathrm{Li}$ atom under an intense laser field to calculate the photoelectron spectra. The computational method has been described in detail in [6,7]. The target Li atom is treated in the single-active-electron approximation by a model potential [26]. The interaction with the linearly polarized laser field is calculated in the dipole length form, and the laser field is assumed to be given by

$$
E_{0} \cos ^{2}\left(\frac{\pi t}{\tau}\right) \cos (\omega t+\varphi),
$$

for $-\tau / 2 \leqslant t \leqslant \tau / 2$, and zero outside of this range. Here the carrier envelope phase is set to $\varphi=\pi / 2$ throughout this work [see Fig. 2(a)]. The peak intensity of the pulse is defined by $I=c \epsilon_{0} E_{0}^{2} / 2$, with $c$ and $\epsilon_{0}$ being the speed of light and the dielectric permittivity, respectively. The pulse duration in terms of full width at half maximum of the intensity is given by $\tau / 2.75$. In the calculations, the pulse duration is $30 \mathrm{fs}$, which is the value used in the experiment of Schuricke et al.

In the numerical solution, the electronic wave function is expanded in spherical coordinates where the radial function is expanded in terms of direct products of discrete variable representation (DVR) functions associated with Legendre polynomials. The time propagation is carried out using the split-operator method in the energy representation of the target atom. We note that a larger time step can be used for longer wavelength lasers in the length form calculations. The photoelectron momentum spectrum is obtained by projecting out the total wave function onto the normalized momentum eigenstate wave functions with the correct boundary conditions at the end of the laser pulse, $t=\tau / 2$.

In actual calculations, the typical radial box size is 2500 a.u., the number of radial grids is 2500 , and the number of time grids is 2000. The angular momenta included in the expansion is from 100 to 500, depending on the laser intensity. Convergence of the calculations has been checked.

\section{IONIZATION MECHANISM AND COMPARISON WITH EXPERIMENTS}

\section{A. Features of strong-field ionization of $\mathrm{Li}$ atom by $785 \mathrm{~nm}$ lasers}

The strong-field ionization of Li by a $785 \mathrm{~nm}$ laser is vastly different from rare-gas atoms because of its much smaller $I_{p}=5.39 \mathrm{eV}$. It takes only four photons to ionize a $\mathrm{Li}$ atom. As stated earlier, at the OBI threshold of $3.4 \mathrm{TW} / \mathrm{cm}^{2}, \gamma=3.6$, which lies in the "conventional" MPI regime. In other words, for the intensity range of 0.4 to $70 \mathrm{TW} / \mathrm{cm}^{2}$ covered by the experiment of Schuricke et al., ionization proceeds from the MPI to OBI regime directly. What are the characteristics of the photoelectron spectrum in the OBI regime? In this regime, the atom is severely ionized before the laser's peak field is reached. Thus the atom is not exposed to the whole pulse duration, i.e., the observed electrons are generated from the leading edge of the pulse only. Indeed, the main features of the experimental spectra of Schuricke et al. hardly change over the whole range of intensities investigated. They are dominated by the four-photon absorption peak, with only a minor presence of the five-photon absorption peak.

\section{B. Multiphoton ionization of $\mathrm{Li}$-total ionization probabilities}

Before comparing with experimental results, first we examine TDSE results for each fixed single peak laser intensity. Figure 1 shows the probabilities for $2 s, 4 f$, sum of Rydberg states (for $n>7$ ), and total ionization as functions of laser intensities. A few notable features are obvious. First, the yields of $2 s$ and $4 f$ oscillate out of phase versus the laser intensity. This is an example of Rabi oscillation between $2 s$ and $4 f$ states via three-photon absorption [27]. For Li, this 


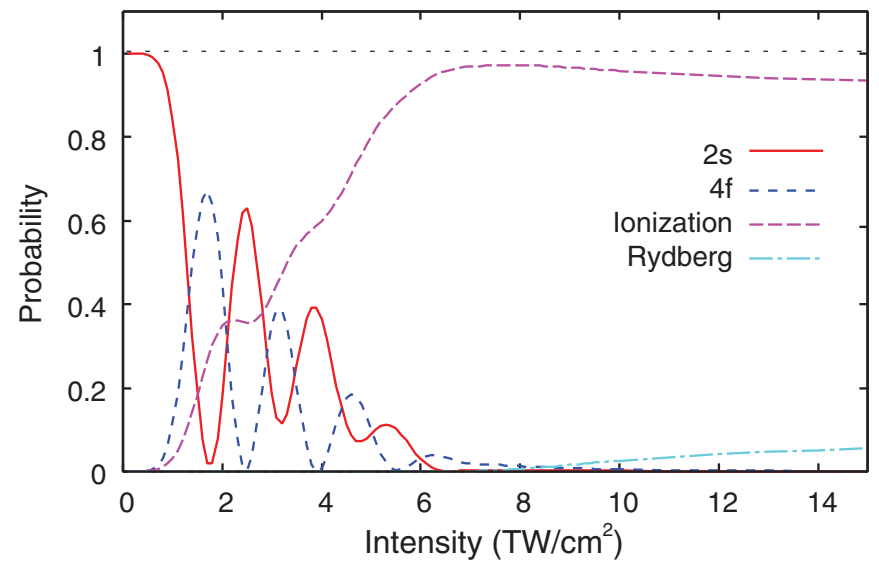

FIG. 1. (Color online) Single intensity excitation and ionization probabilities obtained by solving the TDSE for a $\mathrm{Li}$ atom exposed to a $785 \mathrm{~nm} / 30$ fs laser pulse. Notable features are (1) three-photon Rabi oscillations between $2 s$ and $4 f$, (2) full depletion of the $2 s$ ground state beyond $6 \mathrm{TW} / \mathrm{cm}^{2}$, and of the $4 f$ state beyond $8 \mathrm{TW} / \mathrm{cm}^{2}$, and (3) ionization suppression accompanied by growth of Rydberg states at intensities beyond $8 \mathrm{TW} / \mathrm{cm}^{2}$. Note that atoms are not fully ionized in intense laser fields and survive by staying in Rydberg states.

excitation energy is $4.54 \mathrm{eV}$, which is slightly smaller than the three-photon energy of $4.74 \mathrm{eV}$. As the laser intensity increases, the $4 f$ electron gains additional quiver energy from the laser field such that the $4 f$ state is populated resonantly. At higher intensities, ionization and excitation to other states also occur, thus the out-of-phase oscillations are damped. The total ionization probability increases up to 0.95 with several steps that reflect the $2 s-4 f$ Rabi oscillation until near the $\eta$-OBI threshold of $7.1 \mathrm{TW} / \mathrm{cm}^{2}$, where it begins to flatten out. In the $\eta$-OBI region, a further increase of intensity actually results in a slight decrease of the ionization probability. The total ionization probability never reaches unity. This is an example of ionization suppression, where a few percent of atoms remains neutral, in spite of increasing laser intensity. Note that the decrease of ionization is traced to the increase of Rydberg states. Figure 1 shows that the $2 s$ state is fully depleted above $6.3 \mathrm{TW} / \mathrm{cm}^{2}$ and the $4 f$ state is fully depleted at $8.5 \mathrm{TW} / \mathrm{cm}^{2}$. Thus, at extremely high intensities, neutral atoms exist only in the form of high Rydberg states. These high Rydberg atoms have large induced dipole polarizability. In the presence of an intense laser field, they would experience unprecedented acceleration [28]. The population of Rydberg states will be further analyzed in Sec. V.

To understand the results of Fig. 1, we show in Fig. 2 the time evolution of the "probability" of the unperturbed $2 s$ state within the pulse duration at different intensities. (We comment that this definition is approximately correct only. See [20].) In Fig. 2(a), the electric field of the laser is depicted. Figure 2(b) shows the time evolution for peak intensities below the OBI threshold. At peak intensity of $1.7 \mathrm{TW} / \mathrm{cm}^{2}$, the $2 s$ probability decreases monotonically during the pulse. At $2.5 \mathrm{TW} / \mathrm{cm}^{2}$, the $2 s$ probability drops in the first half of the pulse, but it gains back to reach $60 \%$ at the end of the pulse, reflecting one full Rabi oscillation for the $2 s$. At $3.2 \mathrm{TW} / \mathrm{cm}^{2}$, the $2 s$ population goes through one full Rabi oscillation near the middle of the pulse, but then its probability is lost in the falling tail. From

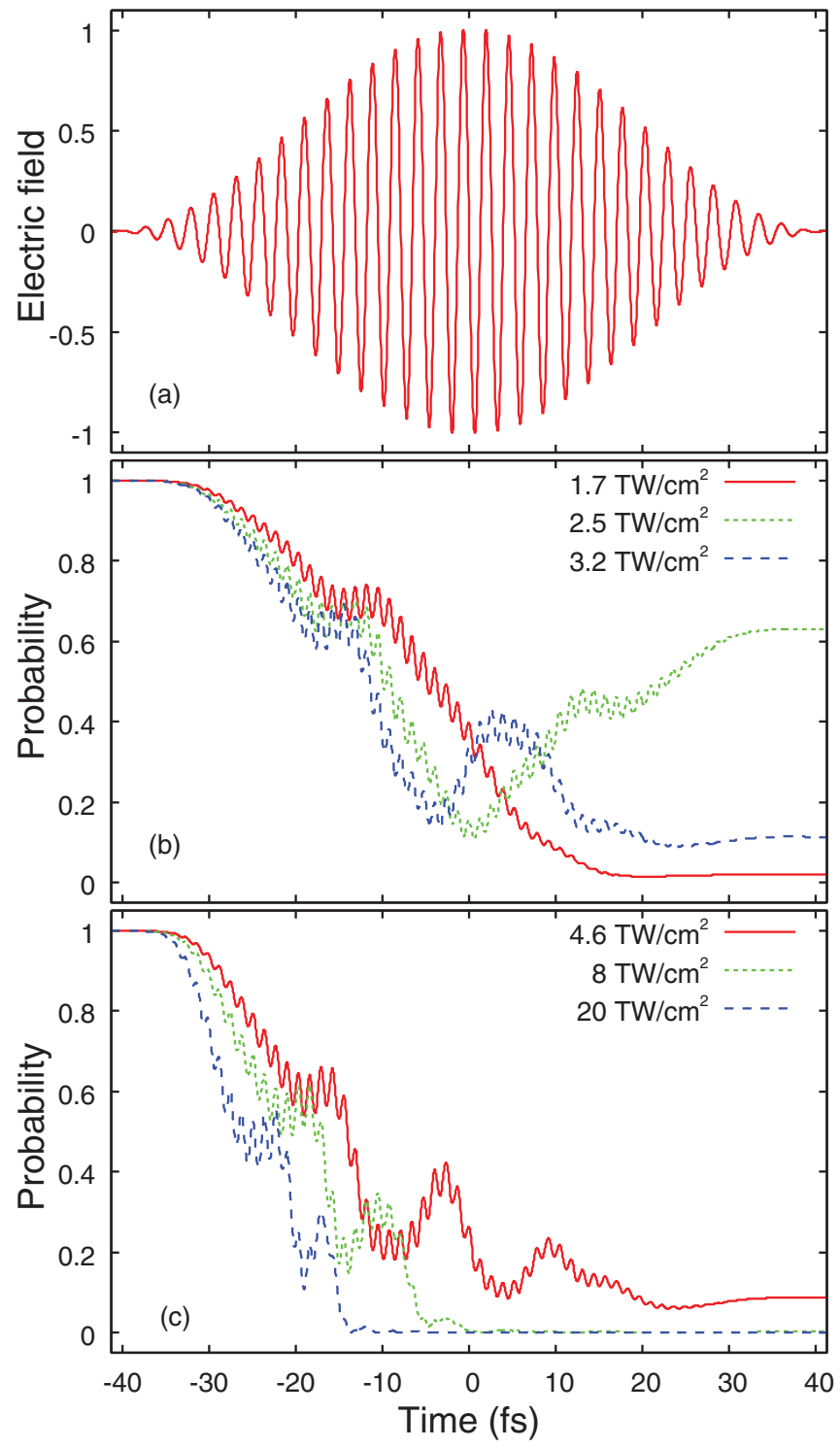

FIG. 2. (Color online) Time dependence of the probability of the field-free $2 s$ state during the laser pulse: (a) Input laser's electric field; (b), (c) $2 s$ survival probability as a function of time for different input intensity.

Fig. 1, it is clear that the probability is lost to the $4 f$ state. Thus, below the OBI threshold of $3.2 \mathrm{TW} / \mathrm{cm}^{2}$, the laser mainly excites Li to the $4 f$ state, with the remaining part in ionization. Figure 2(c) shows the time evolution of $2 s$ probability above the OBI threshold. For $8 \mathrm{TW} / \mathrm{cm}^{2}$, the $2 s$ probability is fully depleted at $t \sim-5 \mathrm{fs}$, while for $20 \mathrm{TW} / \mathrm{cm}^{2}$, it is fully depleted at $t \sim-15$ fs. Thus, at intensities above the OBI threshold, ionization of the atom occurs at the leading edge of the pulse only, not by the full $30 \mathrm{fs}$ pulse. When this happens, it is equivalent that the Li atom is ionized by a short pulse. Thus, the spectral features would be broadened when the peak intensity is raised in the OBI regime.

\section{Effect of integration over laser focus volume}

In strong-field experiments, the laser is focused into a gas cell or a gas jet. Within the focused volume, the laser beam 
intensity has a spatial dependence. To compare with experimental data, integration of yields over the volume has to be carried out [7]. If we assume that the focused volume is smaller than the volume of the gas jet or cell, then the measured photoelectron momentum spectrum $S_{I_{0}}(\mathbf{k})$ can be calculated from

$$
S_{I_{0}}(\mathbf{k}) \propto D \int_{0}^{I_{0}} P(\mathbf{k}, I)\left(-\frac{\partial V}{\partial I}\right) d I,
$$

where $I_{0}$ stands for the peak intensity at the laser focus, $P(\mathbf{k}, I)$ is the photoelectron spectrum calculated with the intensity of $I$, and $D$ is the density of target atoms in the interaction region. Here, $\left(-\frac{\partial V}{\partial I}\right) d I$ represents the differential volume for intensity between $I$ and $I+d I$. We assumed that the spatial intensity distribution is Lorentzian along the propagation direction $(z)$ and Gaussian in the transverse direction [29].

Figure 3 shows the volume-weighted differential ionization yield obtained by summing up the integrand of Eq. (2) over the electron momentum k. In Fig. 3(a), for each $I_{0}$ below the OBI threshold, a range of differential intensities $I$ contributes to the ionization, with the maximal contribution occurring at $I \sim$ $0.75 I_{0}$. Above the OBI threshold, as shown in Fig. 3(b), at each $I_{0}$, the entire peak contribution occurs at $I \sim 1.8 \mathrm{TW} / \mathrm{cm}^{2}$. For higher $I_{0}$, higher $I$ 's have little contributions due to ionization saturation. As demonstrated in Fig. 2(c), for $I \gtrsim 4 \mathrm{TW} / \mathrm{cm}^{2}$, only the early part of the laser pulse contributes to the photoelectron spectra. From Fig. 3(b), one thus expects that photoelectron spectra at all input intensities, $I_{0} \gtrsim 4 \mathrm{TW} / \mathrm{cm}^{2}$
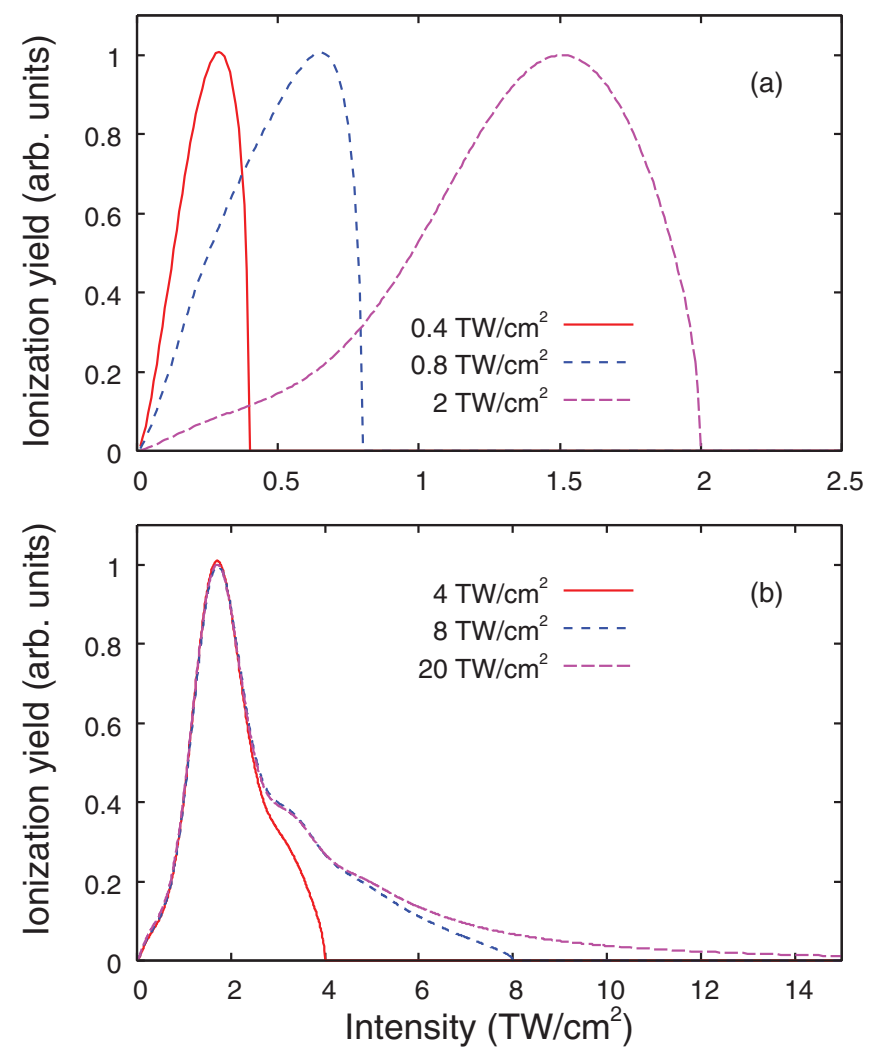

FIG. 3. (Color online) Illustration of the differential intensity contributions to the ionization yield, weighted by the volume element, for a focused laser beam with various peak intensities at the focus. Due to strong depletion, at high peak intensities at the focus, the distributions are very similar.
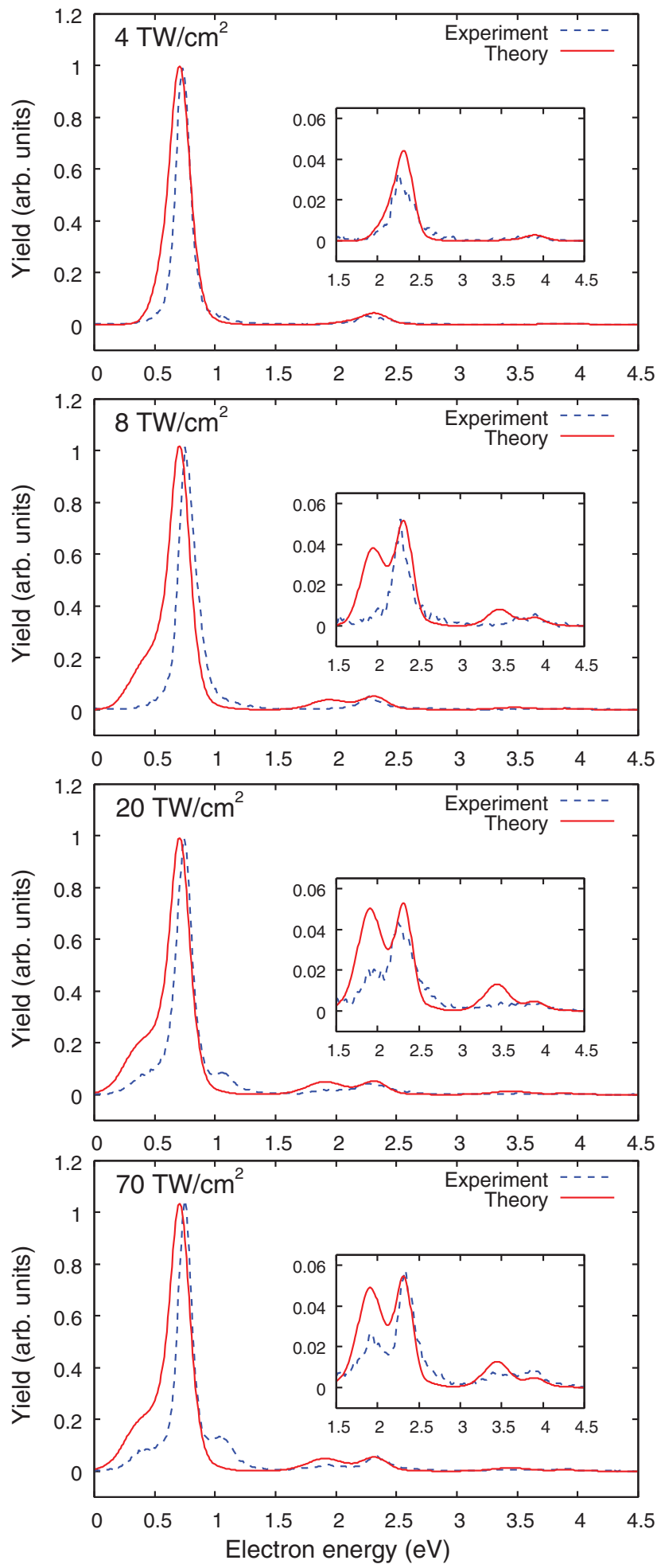

FIG. 4. (Color online) Comparison of theoretical and experimental photoelectron energy spectra at different peak intensities at the laser focus. The experiment is from Schuricke et al. [4]. Note that the photoelectron spectrum is dominated by the four-photon absorption peak, with a minor contribution from the five-photon absorption peak, for intensities from $4-70 \mathrm{TW} / \mathrm{cm}^{2}$. The theory results are from solving the TDSE and include contributions from the whole laser focus volume. The remaining discrepancy is likely due to the fact that parameters for spatial and temporal distributions of the input laser between theory and experiments are not exactly identical. 
would be about the same. Below we compare the calculated spectra with the experimental data of Schuricke $e t$ al.

\section{Comparison with experimental photoelectron energy and two-dimensional momentum spectra}

In Fig. 4, we compare photoelectron energy spectra between theory and experiment at $I_{0}=4,8,20$, and $70 \mathrm{TW} / \mathrm{cm}^{2}$. The spectral features shown in the four frames, for intensities in the OBI regime, are very similar, as expected. In fact, only the four-photon absorption peak near $0.7 \mathrm{eV}$ is clearly seen at all intensities, with a small five-photon absorption peak near $2.3 \mathrm{eV}$ barely visible at higher intensities. However, this does not mean that ionization yield is proportional to the fourth power of intensity. From Fig. 1, the fourth power law is correct only for intensities below $2 \mathrm{TW} / \mathrm{cm}^{2}$. This is the perturbation regime. At higher intensities, the deviation from the fourth power law is partly due to the $2 s-4 f$ three-photon resonance and ionization saturation in the OBI regime. In Fig. 4, the theoretical four-photon absorption peak is consistently broader than the experimental one. Such discrepancy may result from longer pulse duration used in the experiment than in the simulation, but this is probably not the cause since it would need a large error in the reported experimental pulse duration. A more probable explanation may possibly come from the nonuniform gas density distribution in the magnetic-optical trap (MOT). If the density is smaller away from the center, then the high-intensity distribution curves in Fig. 3 will be reduced. This would have the effect of reducing the spread of electron energies, as seen in Fig. 4 (and in Fig. 5). Other possible sources that can contribute to the discrepancy are the laser's spatial and temporal distributions. We note that a large fraction of ionization occurs from the leading edge of the laser, and thus detailed knowledge of the laser pulse in the experiment is essential. There is no reason to expect that the pulse shape used in Eq. (2) is the correct one. For the weak five-photon peaks, their relative values to the four-photon peaks are predicted well by the calculations. At 20 and $70 \mathrm{TW} / \mathrm{cm}^{2}$, the two subpeaks observed are reproduced by the simulation, but the theory predicts much higher strength for the low-energy subpeak.
We next compare in Fig. 5 the two-dimensional (2D) photoelectron momentum spectra, also in the OBI regime only. The horizontal axis is the momentum along the polarization direction, while the vertical axis is the perpendicular component. Only the angular distribution of the four-photon absorption peak is clearly seen; the five-photon absorption peak is barely visible at angles close to the polarization axis. For the four-photon absorption peak, the angular momentum of the photoelectron can have $\ell=0,2$, and 4 . If the absorption went through the $4 f$ state via three-photon resonance, then absorption of one more photon would result in photoelectrons with $\ell=2$ or 4 . The angular distributions in these figures indicate that $\ell=4$ mostly (since the zeros in the distributions are very clear). The width of the ring from the calculation is broader than shown in the experiment, for the possible reasons explained in the energy spectra in the previous paragraph. We have checked that better agreement with experiment is achieved if $50 \%$ of the peak intensity is used in the theoretical calculation. Since the 2D momentum spectra for the four different $I_{0}$ 's differ little, except that the spectral width becomes broader as the input $I_{0}$ is increased, we next set to understand the origin of these broadenings at higher intensities. For this purpose, we analyze the details of the calculated theoretical spectra.

\section{FINE STRUCTURES OF PHOTOELECTRON SPECTRA IN THE OBI REGIME}

In this section, we analyze the photoelectron energy and momentum spectra of Li obtained from solving the TDSE. We treat TDSE results as numerical experimental data and ask whether main features of the calculated spectra can be explained. Figure 6 shows the energy spectra at different laser intensities. Starting with $1.7 \mathrm{TW} / \mathrm{cm}^{2}$, only a four-photon absorption peak is seen clearly. At $3.2 \mathrm{TW} / \mathrm{cm}^{2}$, there are two peaks: one is shifted to the lower energy, while the other stays at the same location. The one at $\sim 0.55 \mathrm{eV}$ is identified to be the nonresonant four-photon absorption peak, which shifts to lower energy as the laser intensity is increased. The second one at $\sim 0.7 \mathrm{eV}$ is the Freeman resonance formed via three-photon absorption to the Stark-shifted $4 f$ state, followed
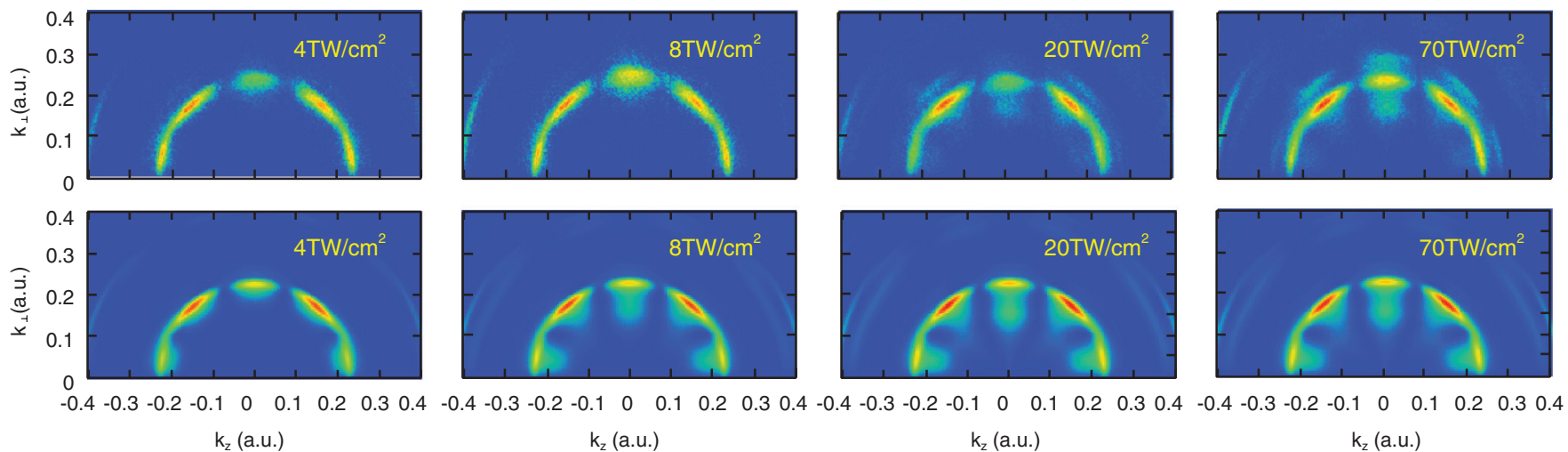

FIG. 5. (Color online) Comparison of experimental (top row) and theoretical (bottom row) 2D momentum spectra for different peak intensities. Note that main features of the angular distributions have not changed over the intensities shown. The theoretical spectra are broader than the experimental data. We found (not shown) that better agreement with experimental data can be seen if the simulation is carried out with half of the indicated experimental peak intensity. Volume effect has been considered in the theoretical calculation. 

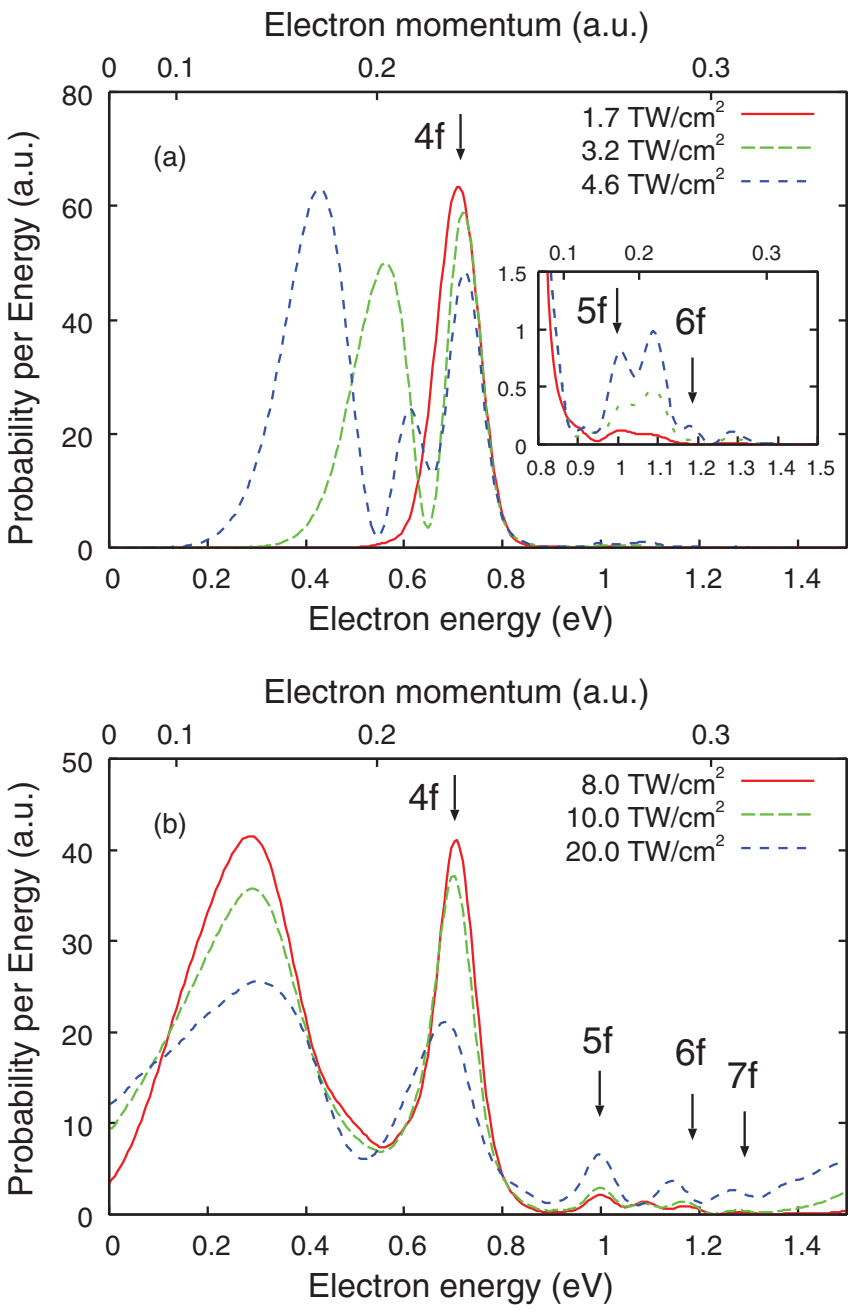

FIG. 6. (Color online) Expanded photoelectron energy spectra at selected intensities. The nonresonant four-photon absorption peak is shown to display the shift to lower energy with increasing intensity, while the main $4 f$ and $5 f$ Freeman resonances are clearly seen in the calculation. Fainter features at higher energies are more difficult to identify. Above $8 \mathrm{TW} / \mathrm{cm}^{2}$, i.e., above the $\eta$-OBI threshold, the four-photon absorption peak position does not change with input peak intensity because of ionization depletion before the pulse is over. In fact, its peak position shifts slightly to higher energy.

by a one-photon absorption to the continuum. Its location is fixed at the one-photon energy of $\hbar \omega$ above the $4 f$ state with respect to the ionization threshold. At $4.6 \mathrm{TW} / \mathrm{cm}^{2}$, three peaks are now seen. The highest energy one again is the $4 f$ Freeman resonance; the lowest one is the nonresonant four-photon absorption peak. The middle weaker one moves rapidly with laser intensity, and we attribute this to be from interference of ionization at different moments of the Rabi oscillation. We continue to the three higher intensities that lie above the OBI threshold; see Fig. 6(b). The $4 f$ Freeman resonance remains at essentially the same position, except that it has been broadened as the peak intensity is increased. For the nonresonant fourphoton absorption peak, its position does not change with peak intensity any more, but its width becomes broader. The fact that the peak positions do not change is consistent with Fig. 3(b), where the equivalent intensity distribution for each

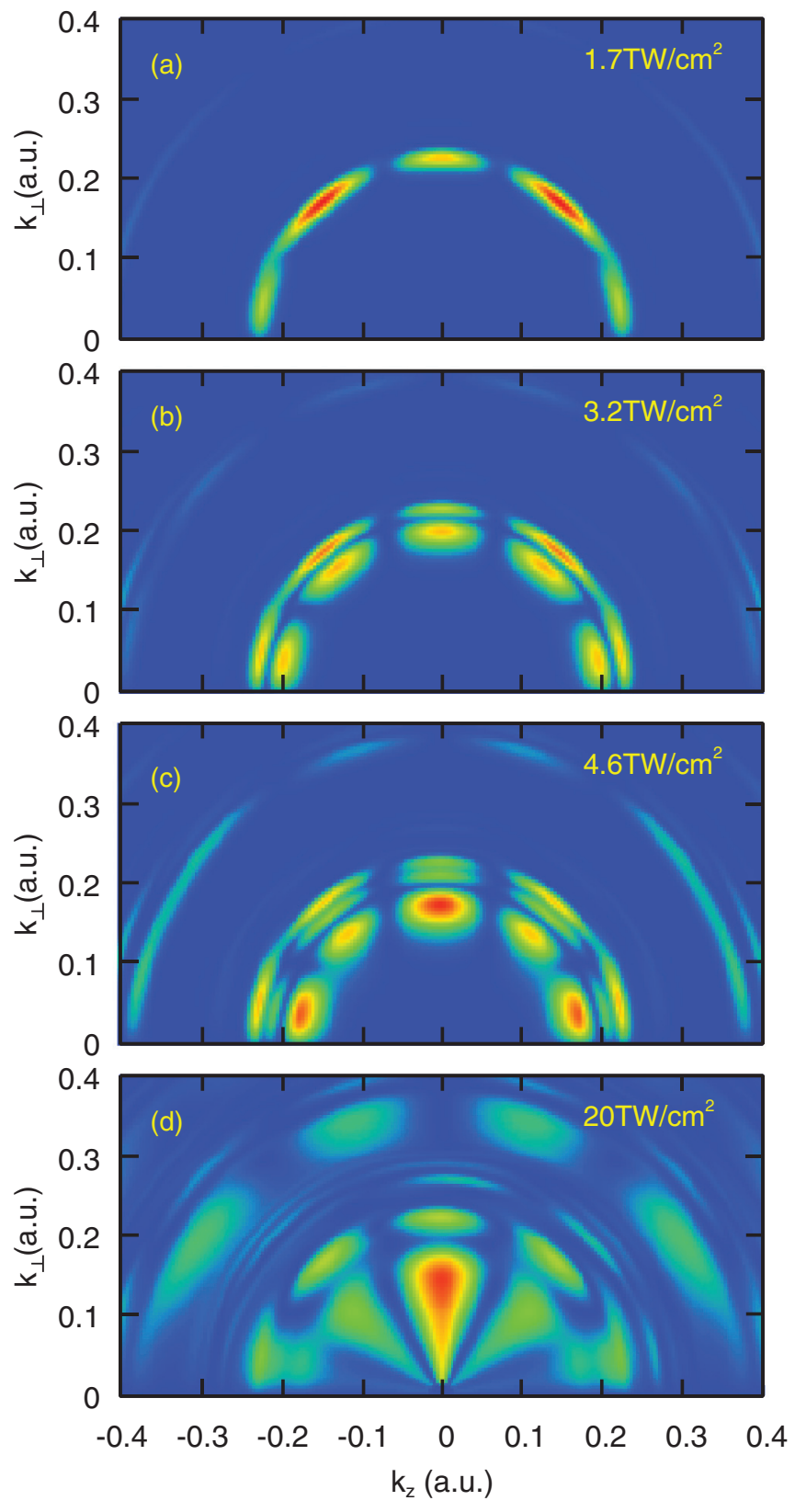

FIG. 7. (Color online) Theoretical 2D momentum spectra at selected intensities shown. Note the presence of split fine structures within the ATI peak.

peak intensity was shown to be identical. Figure 6(a) also shows additional fine spectral features near electron energy at $1.0 \mathrm{eV}$. For clarity, they are shown in expanded scale in the inset. These additional features become much more prominent for higher intensities, as seen in Fig. 6(b). In the figure, the positions of the expected $5 f, 6 f$, and $7 f$ Freeman resonances are also indicated. While the $5 f$ resonance appears to be seen clearly, the others are harder to confirm.

Next, Fig. 7 shows the 2D momentum spectra at different laser intensities. Figure $7\left(\right.$ a) at $I=1.7 \mathrm{TW} / \mathrm{cm}^{2}$ indicates a clear ring at $|k| \sim 0.22$ a.u., associated with the $4 f$ Freeman resonance as shown in Fig. 6(a). As the intensity is increased, as shown in Figs. 7(b)-7(d), the nonresonant ring appears and moves toward lower energies. Note that in spite of the 
spreading due to the depletion of the $2 s$ state for higher intensities, they all have nearly identical angular distributions that are characteristic of four-photon absorption, including the rings at $|k| \sim 0.3$ a.u. corresponding to the $5 f, 6 f$, and $7 f$ resonances in Fig. 6. The five-photon absorption peak, clearly seen at $20 \mathrm{TW} / \mathrm{cm}^{2}$, displays a different angular distribution as expected.

One additional remark should be made as the result of our analysis of the theoretical spectra. In Figs. 6 and 7, the fourphoton absorption peaks were found to move to lower energies with increasing laser intensity. Below $5.0 \mathrm{TW} / \mathrm{cm}^{2}$, the shift is given approximately by $2 U_{p}$, not the commonly used $U_{p}$. If the ac Stark shift of the ground-state level is included, then the theoretical shift would be about $2.1 U_{p}$ [30]. This large shift is due to the fact that the ground state of Li has a large dipole polarizability. Note that both shifts are proportional to the square of the laser-field amplitude or to the laser intensity. In the literature, the lowest peak has been suggested as a possible means to deduce subshell binding energy for complex molecules [31], by measuring electron spectra in strong fields. The accuracy of such a method will be limited in view of uncertainties in laser peak intensities, the presence of Freeman resonances, and the shift of the orbital energy due to induced polarization. For the latter, the energy shift requires knowledge of dynamic polarizability of the subshell.

\section{IONIZATION SUPPRESSION AND RYDBERG STATES IN THE OBI REGIME}

\section{A. The formation of Rydberg states in strong-field ionization of atoms}

Returning to Fig. 1 again, above the $\eta$-OBI threshold, the ionization probability decreases with intensity, while the Rydberg population increases. We can state that this is an example of ionization suppression. The ionization suppression here, however, is not about the removal of the $2 s$ electron, since the $2 s$ electron is already fully depleted at lower intensities. The ionization suppression here is the suppression of ionization of Rydberg states, i.e., the stability of Rydberg states against high-intensity laser fields. Neutral atoms survive extremely intense IR laser fields if they exist as high Rydberg states. Lower excited states can also survive moderately intense laser fields, as seen in earlier experiments [32-36] and in the present calculation for $4 f$ up to about $8 \mathrm{TW} / \mathrm{cm}^{2}$; see Fig. 1 . Based on the numerical calculations carried out here, we will try to offer a mechanism for the formation and stability of high Rydberg states in intense IR laser fields.

In Fig. 8(a), we show the excitation probability of Rydberg states with angular momenta of $\ell=0,2$, and 4 in the $\eta$-OBI regime at $10 \mathrm{TW} / \mathrm{cm}^{2}$. Note that excited states with principal number $n<6$ are not formed. The states with other $\ell$ 's are not
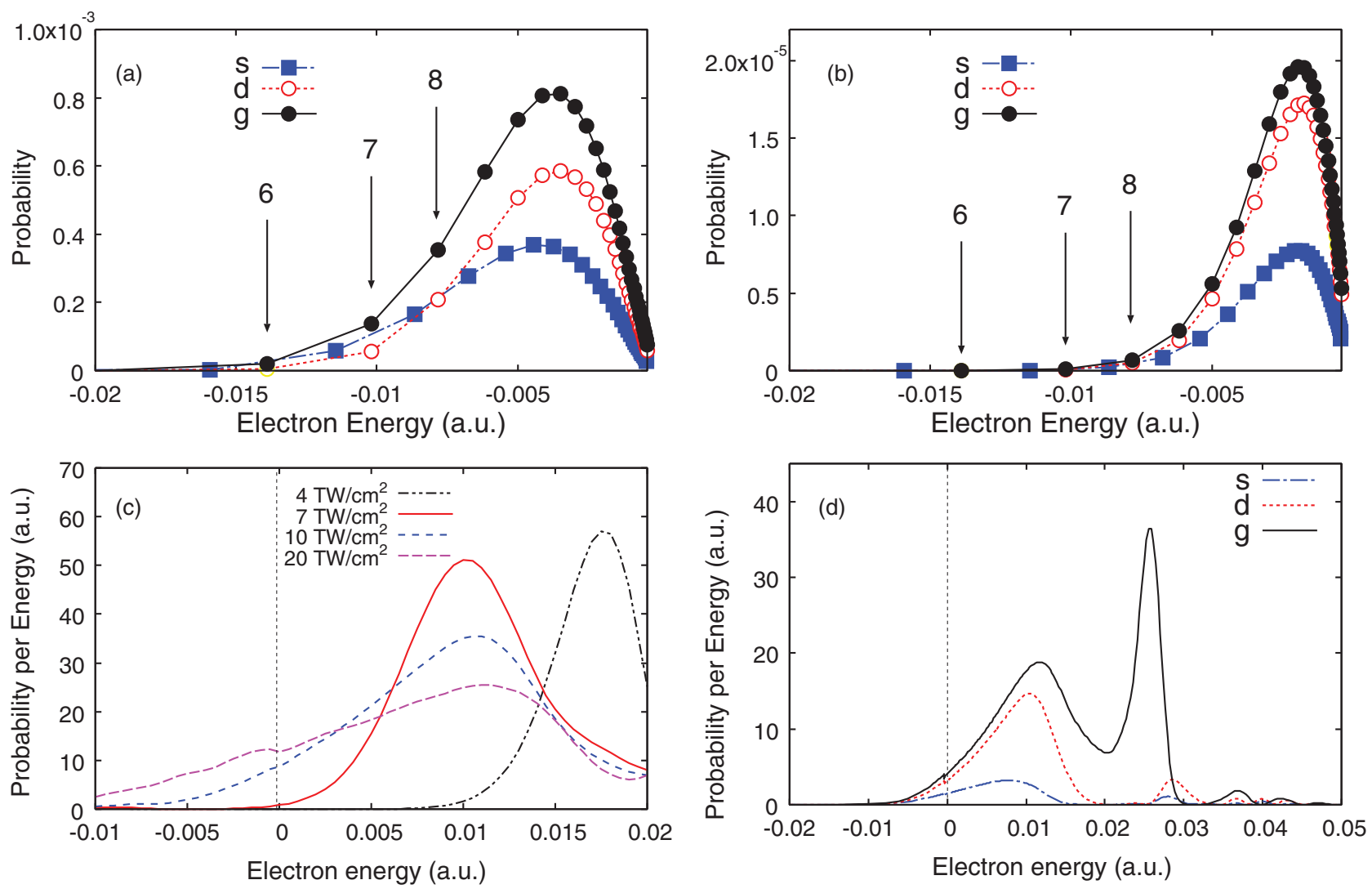

FIG. 8. (Color online) (a) Distributions of high Rydberg states for Li exposed to $30 \mathrm{fs} / 785 \mathrm{~nm}$ laser at intensity of $10 \mathrm{TW} / \mathrm{cm}^{2}$. (b) The same, but at $7 \mathrm{TW} / \mathrm{cm}^{2}$. The arrows and integers indicate the positions of the Rydberg states. (c) Display of excitation probability distributions for Rydberg states and low-energy electrons at several peak intensities. The distributions below the ionization threshold decide the populations of Rydberg states generated by the laser. (d) Population density across the ionization threshold at $10 \mathrm{TW} / \mathrm{cm}^{2}$. A smooth curve is drawn across the threshold for each orbital angular momentum of the electron. 
populated due to selection rules in four-photon absorption. The population peaks at $n \sim 11$. Figure 8 (b) shows the probabilities at the $\eta$-OBI threshold of $7 \mathrm{TW} / \mathrm{cm}^{2}$. The Rydberg state probability peaks at $n \sim 22$, and there are few Rydberg states below $n<8$. Note that the peak Rydberg state probability at $10 \mathrm{TW} / \mathrm{cm}^{2}$ is $5.6 \times 10^{-4}$, while at $7 \mathrm{TW} / \mathrm{cm}^{2}$, it is $2 \times 10^{-5}$, which is a factor of about 30 times smaller.

To understand the general features of the populations of high Rydberg states, it is convenient to express the population in terms of probability density per unit energy, $P_{n} / \Delta E_{n}$, for each partial wave, similar to the one used in [37-39]. With this normalization, the density is smoothly connected to the electron energy spectra across the ionization threshold; see Fig. 8(d). In Fig. 8(c), we show the results at four intensities from 4 to $20 \mathrm{TW} / \mathrm{cm}^{2}$. It is clear that at $4 \mathrm{TW} / \mathrm{cm}^{2}$, the tail of the lower energy side of the four-photon absorption peak is above the threshold, thus no Rydberg states are populated. As intensity is increased from 7 to $20 \mathrm{TW} / \mathrm{cm}^{2}$, the peak becomes broader, with the tail extending below the threshold. Thus, the total Rydberg population increases. This broadening is due to the early depletion of the $2 s$ electron as the laser intensity is increased, and the effect is equivalent to Li ionized by a shorter pulse. This is also consistent with the result shown in Fig. 1, where total Rydberg population increases with intensity in the OBI regime.

Based on the analysis above, we anticipate that the Rydberg population will be enhanced whenever the first ATI peak is close to the ionization threshold. This occurs even at lower laser intensities, below the OBI threshold, at intensities near where channel closing occurs. Suppose an $N$-photon ATI peak appears near the ionization threshold; as the intensity is slightly increased, this $N$-photon ATI peak would have moved down to below the ionization threshold, and the real lowest ATI peak would be due to the $(N+1)$-photon absorption peak, which lies at about $\hbar \omega$ above the ionization threshold. Accordingly, we can anticipate high Rydberg population at each new channel closing. Channel closing does not occur in the present $\mathrm{Li}$ atom under IR laser fields due to its low ionization potential, but would happen many times for a typical $800 \mathrm{~nm}$ laser on a target with higher binding energy. For atomic hydrogen and Ag atoms, earlier TDSE calculations have shown such a result already [40-42]. Clearly, high Rydberg states can also be formed by molecules in intense laser fields. If these Rydberg molecules have repulsive potential curves, they may then dissociate into two or more neutral atoms, thus achieving dissociation without any ionization [43]. A similar observation has also been shown recently for the ionization of molecular ions by intense lasers [44]. We comment that the above analysis is based on a single laser intensity. For a focused laser experiment, the laser intensity is distributed over a broad range, especially in the OBI or the $\eta$-OBI regime, thus high Rydberg states are expected to be observed generally in intense laser experiments.

After analyzing the ubiquitous presence of high Rydberg states of atoms and molecules under an intense laser field, there remain at least two questions:

(1) How are they formed (Q1)?

(2) Why do they survive the intense laser field (Q2)?

These questions have been addressed in the literature in the past: one is the interference stabilization (IS) model [40,41,45], the other is the rescattering (RS) model $[36,46]$. In the RS model, the Rydberg states are formed by recombination of the returning electrons with the ion, at the end of the laser pulse. The RS model does not have to deal with Q2, but this model has been refuted $[41,47]$, since a recombination would have to involve the emission of radiation which was not considered in the RS model according to $[36,46]$. In the IS model, Rydberg states are formed in the early part of the laser pulse (Q1); the survival of Rydberg states in the laser field (Q2) is explained (or modeled) by destructive interference of $\Lambda$-type Raman transitions via the continuum states. This model invokes a second-order process and a destructive interference mechanism to explain the stability of Rydberg states in the intense laser fields. In our interpretation, the answer to Q1 is the same as the IS model: these Rydberg states are formed analogous to the formation of low-lying excited states that are responsible for Freeman resonances. These low-lying excited states are formed via resonant few-photon absorption to ponderomotive energy-shifted excited states. The Freeman resonances seen in the photoelectron spectra are due to absorption of one additional photon from these excited states. In the case of high Rydberg states, their binding energies are very small compared to the photon energy $\hbar \omega$ of an IR laser. Once formed, they are very stable against IR lasers. Such stability is analogous to valence electrons being transparent with respect to $\mathrm{x}$ rays, corresponding to the high-frequency limit of $\epsilon \gg 1$. Our argument of the stability of high Rydberg states against IR lasers is also supported by numerical calculations presented by Potvliege and Smith [48]. These authors showed that the lifetimes of $n=7-8$ Rydberg states for lasers between 455 and $1064 \mathrm{~nm}$ are of the order of more than many tens of picoseconds. The higher the Rydberg states are, the more stable they are against the the same IR laser. Their ionization rates are definitely much smaller than the formation rates since Rydberg states have been observed with lasers of durations of tens to hundreds of femtoseconds. The formation of stable Rydberg states, or population trapping, has also been used by Chin and coworkers [49-51] to explain the bumps observed in the intensity dependence of the total ion yields. Clearly, such small bumps are harder to see for shorter pulses than for longer pulses [49], especially after volume integration over laser intensities in the experiment. Sharper contrast can be easily seen in TDSE calculations carried out at each fixed intensity [40-42,49]. We emphasize that our model of population trapping of Rydberg states does not differ from the earlier model in terms of the formation of Rydberg states, only that we do not rely on the IS mechanism for these states to survive the intense laser field.

Rydberg states would not be formed if circularly polarized IR lasers are used to excite the atom. The reason is similar to the observation that Freeman resonances are not formed in photoelectron spectra if circularly polarized IR lasers are used; see Bucksbaum et al. [52]. Finally, the ellipticity dependence of Rydberg state formation reported in Nubbemeyer et al. [36] has been explained recently by Landsman et al. [47] using semiclassical simulation. They found that these Rydberg atoms are formed by electrons ionized before the peak of the laser field and that rescattering does not play any role. They also used the same theory to explain the lack of low-energy electrons as the ellipticity of the laser is increased, as observed in 
Pfeiffer et al. [53], stressing that the formation of Rydberg states and low-energy photoelectrons are from the same mechanism.

\section{B. Do KH states play a role in strong-field ionization suppression by infrared lasers?}

The theory of ionization suppression of Gavrila and coworkers [22,23], as well as the more recent works [54-56], was formulated in the oscillating frame of the laser field, or in the so-called $\mathrm{KH}$ frame. In this frame, for an atomic potential $V(\mathbf{r})$, the TDSE takes the form

$$
i \frac{\partial}{\partial t} \Psi_{\mathrm{KH}}=\left[-\frac{\nabla^{2}}{2}+V\left(\mathbf{r}+\alpha(t) \mathbf{e}_{z}\right)\right] \Psi_{\mathrm{KH}},
$$

where $\mathbf{e}_{z}$ is the unit vector parallel to the polarization. For a monochromatic laser field $E(t)=E_{0} \cos \omega t$, the ion core executes a harmonic motion, $\alpha(t)=\alpha_{0} \cos \omega t$, where $\alpha_{0}=$ $E_{0} / \omega^{2}$ is the excursion amplitude. Expanding the atomic potential $V$ in a Fourier series in $\omega t$, the leading order term $V_{0}(\mathbf{r})$, called the $\mathrm{KH}$ potential, is independent of time, and supports stationary bound and continuum states. At the high-frequency limit, $V_{0}(\mathbf{r})$ is dominant over all the other higher terms, $V_{n}(n>0)$, and then ionization may be perceived as initiated from the $\mathrm{KH}$ states perturbatively [22]. Under this model, the ionization probability is expected to be small if ionization can be calculated perturbatively. This would require high-frequency fields, or for $\epsilon \gg 1$.

To apply KH-frame formulation to real experiments, the additional issue of how the initial field-free state evolves into $\mathrm{KH}$ states under the laser field with a finite pulse duration will have to be addressed. In a recent paper, Morales et al. [24] claimed that photoelectron momentum spectra for strong-field ionization in the deep OBI regime can be used to image $\mathrm{KH}$ states. They studied the ionization of $\mathrm{K}$ atoms by $800 \mathrm{~nm}$ lasers having a flat top with a few-cycle turn on and turn off. The OBI threshold for K with $I_{p}=4.34 \mathrm{eV}$ is about $1.5 \mathrm{TW} / \mathrm{cm}^{2}$. Their TDSE calculations showed that ionization becomes significant beyond the OBI threshold and stabilization occurs for $\gtrsim 20 \mathrm{TW} / \mathrm{cm}^{2}$, with about $10 \%$ of atoms remaining not ionized at the end of the pulse. Photoelectron momentum spectra were analyzed at two intensities below and above the stabilization intensity, 14 and $56 \mathrm{TW} / \mathrm{cm}^{2}$. They found that for both intensities, the momentum spectra calculated from the standard TDSE method can be reproduced fairly well by calculations carried out in the $\mathrm{KH}$ frame, with the absorption of a few photons, assuming that the electron is initially in the $\mathrm{KH}$ states where the ground $4 s$ and excited $4 p$ states are strongly coupled with the $800 \mathrm{~nm}$ field. Based on such an observation, they concluded that photoelectron momentum spectra in the OBI regime can be used to image KH states.

The $\mathrm{Li}$ atom studied in this article is very similar to the $\mathrm{K}$ atom investigated in Morales et al. For the Li case, $2 s$ and $4 f$ states are strongly coupled by the three-photon absorption. In our calculations, stabilization occurs in the OBI regime, which is similar to the $\mathrm{K}$ case in Morales et al. As shown in Figs. 6 and 7, below the $\eta$-OBI threshold and before stabilization occurs, the ATI peaks move to lower energies with increasing laser intensity. The shift of the energy position of the ATI peaks can be approximated by the two comparable contributions from the shift of the ground-state energy and the ponderomotive shift of the ionization threshold. In the deep stabilization regime above the $\eta$-OBI threshold, the ATI peak does not change with input laser intensities. However, it is not clear how the ATI peak shift is to be interpreted in the $\mathrm{KH}$ picture. For lower intensities below the $\eta$-OBI threshold, the energy shift of the $\mathrm{KH}$ ground state is identical to the ac Stark shift due to the dynamical dipole polarizability. But the ponderomotive shift would not be described by a few harmonics in the $\mathrm{KH}$ potentials, since the coupling among the continuum states is very weak. In addition, for higher intensities in the $\eta$-OBI regime, features of photoelectron energy and momentum spectra can be easily understood in terms of depletion of the $2 s$ state that occurs at the leading edge of the laser pulse, as we have shown in this work. Before the peak laser field is reached, there is no $2 s$ electron remaining in the atom, and there is no evidence that $\mathrm{KH}$ states associated with the $2 s$ state are formed in the laser field, since these states would be much more localized than the remaining high Rydberg states in the atom found in the TDSE calculations.

In our opinion, $\mathrm{KH}$-frame formulation is relevant only in the high-frequency limit of $\epsilon \gg 1$ with slowly varying envelope of the pulse, where higher order terms can be neglected [54,55]. Such conditions are satisfied for ionization of Rydberg states by IR lasers, or by simple atomic hydrogen or helium in the field of intense $x$ rays. The latter may become available in the near future with the emergence of free-electron x-ray lasers. The ionization suppression or stabilization observed in the present system is the stability of Rydberg states against intense IR pulses. We conclude that KH states play no role in the ionization of $\mathrm{Li}$ or $\mathrm{K}$ atoms using $800 \mathrm{~nm}$ lasers where $\epsilon$ is close to $0.3-0.4$ only.

\section{SUMMARY AND DISCUSSION}

In this article, we examined strong-field ionization of $\mathrm{Li}$ atoms by an intense $785 \mathrm{~nm}$ laser at intensities up to $70 \mathrm{TW} / \mathrm{cm}^{2}$. Our initial goal was to obtain photoelectron spectra by solving the time-dependent Schrödinger equation to compare with the experimental data of Schuricke et al. Since the static over-the-barrier ionization (OBI) threshold intensity for $\mathrm{Li}$ is only $3.4 \mathrm{TW} / \mathrm{cm}^{2}$, most of the experimental data were taken in the OBI regime. At intensities way over the OBI threshold, the $2 s$ electron is fully depleted before the peak intensity of the pulse is reached. The recorded photoelectron momentum spectra are generated from the early part of the pulse, and are nearly independent of input laser intensity. The agreement between simulation and experiment is considered to be good in view of the fact that detailed experimental conditions are not fully known.

Ionization suppression has been observed in our calculations at intensities way above the OBI threshold. This was traced to the presence of high Rydberg states, which are stable against ionization by the infrared lasers. We studied the mechanism of the formation and stability of these Rydberg states. Our mechanism differs from the 
well-accepted interference stabilization model for the trapping of Rydberg states, and also from the rescattering model proposed recently. In addition, we also disagree with the recent interpretation that one can image the so-called $\mathrm{KH}$ states by measuring photoelectron momentum spectra at extreme high intensities using infrared lasers for atoms and molecules.

\section{ACKNOWLEDGMENTS}

This work was supported in part by the Grants-in-Aid for scientific research (B) and (C) from the Japan Society for the Promotion of Science and by the Chemical Sciences, Geosciences and Biosciences Division, Office of Basic Energy Sciences, Office of Science, US Department of Energy. T.M. thanks O. I. Tolstikhin for fruitful discussion.
[1] A. Rudenko, K. Zrost, C. D. Schröter, V. L. B. de Jesus, B. Feuerstein, R. Moshammer, and J. Ullrich, J. Phys. B 37, L407 (2004).

[2] C. M. Maharjan, A. S. Alnaser, I. Litvinyuk, P. Ranitovic, and C. L. Cocke, J. Phys. B 39, 1955 (2006).

[3] T. Marchenko, H. G. Muller, K. J. Schafer, and M. J. J. Vrakking, J. Phys. B 43, 185001 (2010).

[4] M. Schuricke, G. Zhu, J. Steinmann, K. Simeonidis, I. Ivanov, A. Kheifets, A. N. Grum-Grzhimailo, K. Bartschat, A. Dorn, and J. Ullrich, Phys. Rev. A 83, 023413 (2011).

[5] M. Wickenhauser, X. M. Tong, D. G. Arbó, J. Burgdörfer, and C. D. Lin, Phys. Rev. A 74, 041402 (2006).

[6] Z. Chen, T. Morishita, A. T. Le, M. Wickenhauser, X. M. Tong, and C. D. Lin, Phys. Rev. A 74, 053405 (2006).

[7] T. Morishita, Z. Chen, S. Watanabe, and C. D. Lin, Phys. Rev. A 75, 023407 (2007).

[8] M. Abu-Samha and L. B. Madsen, J. Phys. B 44, 235601 (2011).

[9] D. G. Arbó, J. E. Miraglia, M. S. Gravielle, K. Schiessl, E. Persson, and J. Burgdörfer, Phys. Rev. A 77, 013401 (2008).

[10] P. Colosimo et al., Nature Phys. 5, 335 (2009).

[11] W. Quan, Z. Lin, M. Wu, H. Kang, H. Liu, X. Liu, J. Chen, J. Liu, X. T. He, S. G. Chen, H. Xiong, L. Guo, H. Xu, Y. Fu, Y. Cheng, and Z. Z. Xu, Phys. Rev. Lett. 103, 093001 (2009).

[12] C. Y. Wu, Y. D. Yang, Y. Q. Liu, Q. H. Gong, M. Y. Wu, X. Liu, X. L. Hao, W. D. Li, X. T. He, and J. Chen, Phys. Rev. Lett. 109, 043001 (2012)

[13] G. G. Paulus, W. Becker, W. Nicklich, and H. Walther, J. Phys. B 27, L703 (1994).

[14] T. Morishita, A.-T. Le, Z. Chen, and C. D. Lin, Phys. Rev. Lett. 100, 013903 (2008).

[15] P. B. Corkum, Phys. Rev. Lett. 71, 1994 (1993).

[16] K. J. Schafer, B. Yang, L. F. DiMauro, and K. C. Kulander, Phys. Rev. Lett. 70, 1599 (1993).

[17] C. D. Lin, A. T. Le, Z. Chen, T. Morishita, and R. Lucchese, J. Phys. B 43, 122001 (2010).

[18] L. Hamonou, T. Morishita, and O. I. Tolstikhin, Phys. Rev. A 86, 013412 (2012).

[19] O. I. Tolstikhin and T. Morishita, Phys. Rev. A 86, 043417 (2012).

[20] O. I. Tolstikhin, T. Morishita, and S. Watanabe, Phys. Rev. A 81, 033415 (2010).

[21] Y. Okajima, O. I. Tolstikhin, and T. Morishita, Phys. Rev. A 85, 063406 (2012).

[22] M. Gavrila, J. Phys. B 35, R147 (2002).

[23] M. Pont and M. Gavrila, Phys. Rev. Lett. 65, 2362 (1990).

[24] F. Morales, M. Richter, S. Patchkovskii, and O. Smirnova, Proc. Natl. Acad. Sci. USA 44, 101003 (2012).
[25] S.-D. Jheng and T. F. Jiang, J. Phys. B 46, 115601 (2013).

[26] W. Shweizer and P. Fassbunfer, At. Data Nucl. Data Tables 72, 33 (1999).

[27] A. F. Linskens, I. Holleman, N. Dam, and J. Reuss, Phys. Rev. A 54, 4854 (1996).

[28] U. Eichmann, T. Nubbemeyer, H. Rottke, and W. Sandner, Nature (London) 461, 1261 (2009).

[29] S. Augst, D. D. Meyerhofer, D. Strickland, and S. L. Chin, J. Opt. Soc. Am. B 8, 858 (1991).

[30] The dipole polarizability of an Li atom is quite large. Using the value of $\alpha=352$ a.u. at $\omega=0.05$ a.u. from M. Merawa and M. Rerat, J. Chem. Phys. 108, 7060 (1998), the calculated energy shift of the ground state is $1.13 U_{p}$. This would give a $2.13 U_{p}$ for the ATI peak, close to the empirical shift of $2.0 U_{p}$ found from the calculated photoelectron spectra. If the static dipolarizability of 164 a.u. were used, the ground-state shift would be $0.53 U_{p}$.

[31] A. Boguslavskiy, J. Mikosch, A. Gijsbertsen, M. Spanner, S. Patchkovskii, N. Gador, M. Vrakking, and A. Stolow, Science 335, 1336 (2012).

[32] M. P. de Boer, J. H. Hoogenraad, R. B. Vrijen, L. D. Noordam, and H. G. Muller, Phys. Rev. Lett. 71, 3263 (1993).

[33] J. H. Hoogenraad, R. B. Vrijen, and L. D. Noordam, Phys. Rev. A 50, 4133 (1994).

[34] M. P. deBoer and H. G. Muller, Phys. Rev. Lett. 68, 2747 (1992).

[35] R. R. Jones, D. W. Schumacher, and P. H. Bucksbaum, Phys. Rev. A 47, R49 (1993).

[36] T. Nubbemeyer, K. Gorling, A. Saenz, U. Eichmann, and W. Sandner, Phys. Rev. Lett. 101, 233001 (2008).

[37] H. Friedrich, Theoretical Atomic Physics (Springer, Berlin, 1991), p. 128.

[38] C. D. Lin, Astrop. J. 187, 385 (1974).

[39] C. N. Liu, A. Hishikawa, and T. Morishita, Phys. Rev. A 86, 053426 (2012).

[40] A. M. Popov, O. V. Tikhonova, and E. A. Volkova, J. Mod. Opt. 58, 1195 (2011).

[41] E. A. Volkova, A. M. Popov, and O. V. Tikhonova, J. Exp. Theor. Phys. 113, 394 (2011).

[42] A. M. Popov, O. V. Tikhonova, and E. A. Volkova, Laser Phys. 20, 1028 (2010).

[43] B. Manschwetus, T. Nubbemeyer, K. Gorling, G. Steinmeyer, U. Eichmann, H. Rottke, and W. Sandner, Phys. Rev. Lett. 102, 113002 (2009).

[44] J. McKenna, S. Zeng, J. J. Hua, A. M. Sayler, M. Zohrabi, Nora G. Johnson, B. Gaire, K. D. Carnes, B. D. Esry, and I. Ben-Itzhak, Phys. Rev. A 84, 043425 (2011).

[45] M. Fedorov and A. Movsesian, J. Phys. B 21, L155 (1988).

[46] N. Shetsov-Shilovski, S. Goreslavski, S. Popruzhenko, and W. Becker, Laser Phys. 19, 1550 (2009). 
[47] A. S. Landsman, A. N. Pfeiffer, C. Hofmann, M. Smolarski, C. Cirelli, and U. Keller, New J. Phys. 15, 013001 (2013).

[48] R. M. Potvliege and P. H. G. Smith, Phys. Rev. A 48, R46 (1993).

[49] A. Azarm et al., J. Phys: Conf. Ser. 414, 012015 (2013).

[50] A. Talebpour, Y. Liang, and S. L. Chin, J. Phys. B 29, 3435 (1996).

[51] A. Talebpour, C. Y. Chien, and S. L. Chin, J. Phys. B 29, 5725 (1996).

[52] P. H. Bucksbaum, L. D. Van Woerkom, R. R. Freeman, and D. W. Schumacher, Phys. Rev. A 41, 4119 (1990).
[53] A. N. Pfeiffer, C. Cirelli, A. S. Landsman, M. Smolarski, D. Dimitrovski, L. B. Madsen, and U. Keller, Phys. Rev. Lett. 109, 083002 (2012).

[54] K. Toyota, O. I. Tolstikhin, T. Morishita, and S. Watanabe, Phys. Rev. A 76, 043418 (2007).

[55] K. Toyota, O. I. Tolstikhin, T. Morishita, and S. Watanabe, Phys. Rev. A 78, 033432 (2008).

[56] K. Toyota, O. I. Tolstikhin, T. Morishita, and S. Watanabe, Phys. Rev. Lett. 103, 153003 (2009). 